Apidologie, 1983, 14 (2), 105-118

\title{
MESURE DE LA COULEUR DES MIELS
}

\author{
Serge AUBERT* et Michel GONNET** \\ * I.N.R.A., Station de technologie, Domaine Saint-Paul, 84140 Montfavet \\ ** I.N.R.A., Station de zoologie (apiculture), Domaine Saint-Paul, 84140 Montfavet
}

RÉSUMÉ

La coloration de miels typiques a été étudiee à l'aide de la méthodologie tristimulaire d'analyse spectrophotométrique adoptée par la C.I.E. (Convention Internationale de l'Éclairage). Ces coordonnées chromatiques, objectives et précises, sont confrontées avec les données d'un comparateur visuel classique (système Pfund Lovibond).

Cette méthode permet d'effectuer le classement précis de vingt miels d'origines florales différentes en fonction de la couleur. Le classement par simple appréciation visuelle est très subjectif et erroné.

Les comparateurs colorimétriques usuels existants sur le marché (mesures en indice de Pfund) sont justifiés pour une utilisation commerciale courante, ils ne suffisent pas pour séparer des miels originaux très clairs, très foncés ou opaques et cristallisés.

\section{I. - AGRÉMENT VISUEL ET APPELLATION DES MIELS}

La couleur d'un produit alimentaire est un facteur d'attrait commercial important : elle guide très souvent le choix du consommateur. Celui-ci réagit à la stimulation visuelle par des réactions plus ou moins rationnelles, dans une analyse psycho-sensorielle sommaire. La notion de qualité pour les consommateurs est généralement liée à la connaissance traditionnelle, analogique, qu'il a d'une denrée.

Ainsi, un miel très foncé est subjectivement associé à un produit fortement aromatique, alors qu'un miel clair suggère des arômes plus fins, plus subtils. Bien que ce phénomène se vérifie souvent; il n'est pas la règle absolue. Les rapports entre la couleur et l'arôme des miels restent aléatoires. De même, un miel liquide de couleur vive, brillante, agréable à l'œil, peut être préféré, alors qu'en concurrence un produit tout à fait identique, mais en phase cristallisé, donnant une apparence plus claire et plus terne, sera écarté. Le choix au niveau du critère texture et agrément d'utilisation (aptitude à tartiner) relève aussi d'une appréciation tactile; il y a les amateurs du miel 
liquide et les fervents du miel cristallisé. Pour une majorité de profanes souvent moins motivés dans leurs préférences, la couleur du produit devient cependant le critère déterminant.

Dans un état physique stable, liquide ou cristallisé, un miel provenant d'une source végétale définie doit avoir une coloration conforme à celle attendue et reconnue par le consommateur averti. Ainsi, un miel d'acacia ne se vend bien que s'il est clair et limpide; un miel de callune doit être brun roussâtre et " bullé "; un miel de miellat de sapin des Vosges est particulièrement apprécié avec une coloration presque noire et des reflets verdâtres. Pour les miels multifloraux, les normes de couleur restent imprécises, mais il est toujours souhaitable de retrouver, chaque année, une coloration constante dans sa tonalité et luminosité moyenne, en accord avec l'appréciation coutumière d'un certain type de production.

Pour y réussir, on peut être amené à faire des mélanges de miels. Ils seront confectionnés sur des bases florales définies, tout en respectant ou en recherchant une norme de couleur habituelle ou sélectionnée. Pour répondre à ces problèmes d'adaptation aux goûts diversifiés des consommateurs, il faut disposer de références objectives, précises et reproductibles.

\section{II. - TECHNIQUES ACTUELLES DE MESURE DE LA COULEUR DES MIELS ET LEURS LIMITES}

La mesure de la couleur des miels a fait l'objet de divers travaux, plus ou moins empiriques et anciens. II est nécessaire d'actualiser nos connaissances et d'apporter des solutions nouvelles à ce problème de la qualité visuelle.

Dès le début du siècle, les apiculteurs utilisaient pour référencer leur miel des plaques de verre teintées : blanches, jaunes et brunes. Le miel était introduit dans de petites bouteilles plates et l'on comparait les échantillons aux plaques étalons numérotées. Cette gamme conventionnelle, empirique et très arbitraire, a été commercialisée en Europe sous l'appellation discutable de "melloscope universel ».

Aux États-Unis, Sechrist (1925) propose un comparateur visuel plus perfectionné appelé "Pfundcolor grader ". Cet appareil est équipé d'un filtre en verre teinté (caramel) en forme de coin très fermé. L’intensité de la couleur mesurée varie en fonction de l'épaisseur du prisme coloré, situé dans le même champ optique. Juste au-dessus du filtre prismatique une cuve de forme identique reçoit le miel. Ces deux coins superposés, en opposition, sont déplacés ensemble sur un chariot le long d'une règle graduée. La source lumineuse placée à larrière est observée à travers deux fenêtres fixes superposées : l'une ouverte sur le prisme de verre coloré. l'autre sur la cuve contenant le miel. On réalise l'égalité de coloration entre les deux niveaux d'ouverture. La lecture correspond à la distance du déplacement du chariot. Elle s'exprime en centimètres et en millimètres. Cet "indice de Pfund» se situe autour de : une unité pour les miels les plus clairs el de quatorze unités pour les plus foncés. L'auteur défini, avec cette échelle, sept colorations croissantes conventionnelles. Cette classification est admise officiellement dans le domaine commercial en Amérique du Nord : Blanc d'eau, Extra-blanc, Blanc, Ambré extra clair, Ambré clair, Ambré "l Foncé.

Toujours aux États-Unis BRICE et al (1956) ont mis au point un appareil usuel, très simple, fondé sur le système Pfund. Six filtres de verre sont étalonnés, par comparaison au spectrophotomètre, avec des solutions conventionnelles de caramel dans de la glycérine. Des suspensions aqueuses de bentonite, logées en récipients plats, sont placées sur un portoir derrière les filtres; elles permettent de simuler un trouble 
plus ou moins accentué. Le miel à caractériser, mis dans une cuve plate aux dimensions définies, est comparé aux différents filtres étalons. Cela ressemble au "melloscope", mais codifié plus rigoureusement.

La comparaison à d'autres gammes étalons objectives fut la préoccupation de nombreux auteurs. Citons l'étalon de référence colorimétrique de BARBIER et VALIN (1957). lls utilisent des solution iode/iodure de normalités croissantes. Cette gamme de couleur est concrétisée sur 5 étalons recouvrant sensiblement l'ensemble de la palette chromatique des miels. La présence d'un trouble peut être simulée à l'aide d'écrans au sulfate de baryum en suspension dans l'eau. Les solutions étalons d'iode doivent être refaites fréquemment, leur couleur faiblissant rapidement.

Actuellement, les techniques de référence internationale toujours en vigueur pour définir la couleur des miels sont fondées sur la méthode de Pfund. Les mesures précises sont pratiquées : soit directement avec le montage du «Pfund color grader », soit à l'aide d'un comparateur de type Lovibond. Ce dernier appareil, plus compact, est équipé de deux disques chromatiques; l'un pour les miels clairs, l'autre pour les miels foncés. Chaque disque comporte neuf pastilles de verre coloré d'intensité croissante étalonnées sur les références de Pfund. Le miel liquide est observé dans une cuve carrée de 10 millimètres de trajet optique. On fait défiler la gamme colorée du disque choisi à côté de la cuve à échantillon. Quand la couleur observée au niveau des deux compartiments est d'égale intensité, on note le numéro de la pastille correspondante. Pour réaliser l'égalité des plages, le comparateur peut être placé face à une source lumineuse naturelle ou, comme sur la figure 1, il peut être enfermé dans un boîtier métallique en avant d'une source lumineuse artificielle (lampe type « lumière de jour »). Les résultats sont traduits en « indice de Pfund $»$.

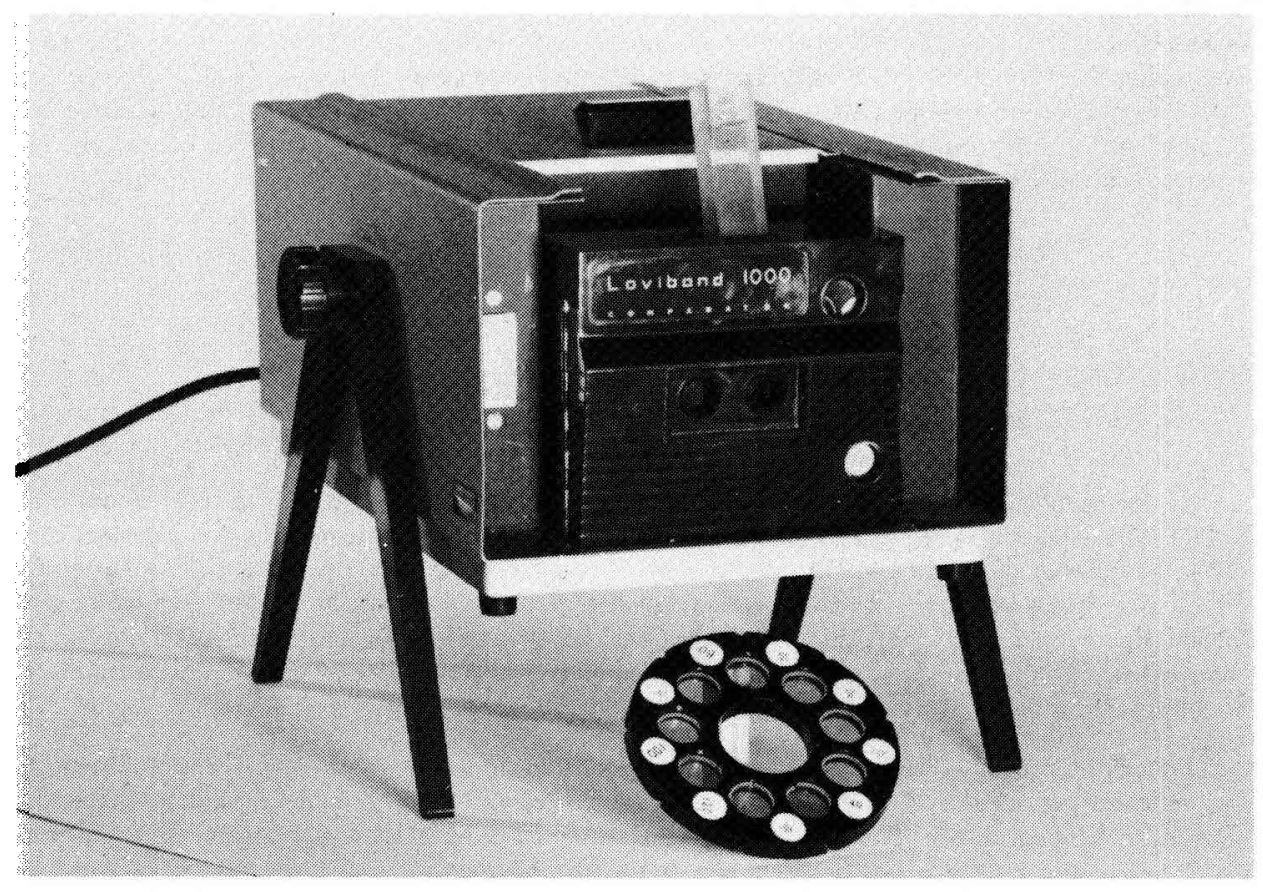

FIG. 1. - Comparateur visuel de LoviBOND

Deux disques comportent chacun neuf pastilles colorées et référencées.

FIG. 1. - Visual LoviBOND comparator.

The two discs contain nine color and reference patches. 
Néanmoins. toutes les techniques mises en ceuvre pour caractériser la couleur des miels sont pratiquées sur des produits à l'ètat liquide. Les miels cristallisés doivent donc subir une refonte préalable. Les miels en phase liquide sont généralement homogènes et parfois limpides. La spectrophotométrie, par lecture directe sous une ou plusieurs longueur d'onde prédéterminées, serait une méthode d'investigation à première vue bien adaptée au problème. SECHRIST (1925) a démontré le caractère imprécis et aléatoire des résultats obtenus sur une cellule photo électrique de mesure dont le signal ne distingue pas la part respective de l'absorption lumineuse due au trouble de celle venant de la pigmentation vraie. Selon les références américaines, un miel "Blanc " accusant un léger trouble donne, sur longueur d’onde déterminée, une densité optique équivalente ou supérieure à celle d'un miel " Ambré extra clair ", mais limpide. De plus, la longueur d'onde optimale à utiliser pour la mesure varie sensiblement d'un miel à l'autre selon sa tonalité. Dans ces conditions, il est bien difficile d'obtenir des résultats d'ensemble cohérents et reproductibles.

Pour l'étalonnage des verres colorés (à l'aide des substances de référence : caramel + glycérine), BRICE et al.(1956) ont utilisé une technique spectrophotométrique s inspirant de la méthodologie dite «tristimulaire ». Cette méthode, fondée sur la trivariance de la couleur, a été officialisée par la Commission Internationale de l'Éclairage (C.I.E.); ce qui lui donne un caractère plus « universel » et surtout fondamentalement liée à une perception visuelle humaine « standardisée ". Cette voie d'approche, plus objective, ne dépendant pas de façon aléatoire de chaque observateur, offre plus de rigueur dans les mesures cherchant à préciser la palette de couleur des miels. Nous avons adopté cette méthodologie moderne, déjà appliquée sur de nombreuses denrées végétales et animales (AUBERT, 1976). Dans notre travail, les mesures portent directement sur des miels de différentes origines et non pas sur des solutions pures de référence. comme l'ont fait les auteurs américains. En effet, toutes ces dilutions de caramel, de la plus claire à la plus foncée, ont la même tonalité de couleur. Il est bien évident qu'une telle homogénéité de pigmentation ne se retrouve pas entre tous les miels d'origines diverses. Les objectifs visés dans cette recherche sont : d'une part, une meilleure définition des couleurs mesurées et d'autre part, une correction éventuelle des techniques usuelles utilisant des comparateurs de type «Pfund ». En effet, la cohérence et les limites des comparateurs visuels simples sont à préciser. L'utilisation d'une méthode plus universelle dappréciation de la couleur perçue par tous les consommateurs doit permettre un classement plus rigoureux des miels, notamment dans la définition des produits à appellations.

\section{III. - MATÉRIEL ET MÉTHODES PROPOSÉES}

La méthodologie adoptée est aujourd'hui classique (AUBERT S., 1976) et utilisée sur de nombreux produits alimentaires liquides, solides et opaques, à l'aide d'appareils de réflectométrie. Son principe repose sur la trivariance des couleurs. On adopte trois couleurs de référence (bleu, vert et rouge) et une représentation spatiale à trois dimensions dans un système mathématique permettant de projeter toute couleur sur un plan et à l'intérieur d'un triangle de chromaticité (qualité de la couleur). Le calcul des coordonnées chromatiques et les déterminations graphiques ont été réalisées d'après le livre « Handbook of colorimetry " de HARDY (1936).

Les calculs peuvent s'effectuer manuellement, sur les tracés spectrographiques, par la technique des ordonnées sélectionnées de HARDY, plus ou moins simplifiées (10 ou 100 mesures par stimulus). Actuellement, cette exploitation des données colorimétriques se fait en utilisant toutes les ressources de l'informatique (microordinateur et table traçante) (Fig. 2).

Les déterminations portent sur un large éventail de vingt échantillons de miels sélectionnés de qualité florale différente. Ils sont présentés dans les tabieaux en tenant compte de l'ordre de classement visuel et empirique sur la couleur. Ce tri subjectif s'effectue en observant les miels sous une même épaisseur. On les classe en allant du plus clair au plus foncé en précisant ensuite lorigine florale correspondante. 


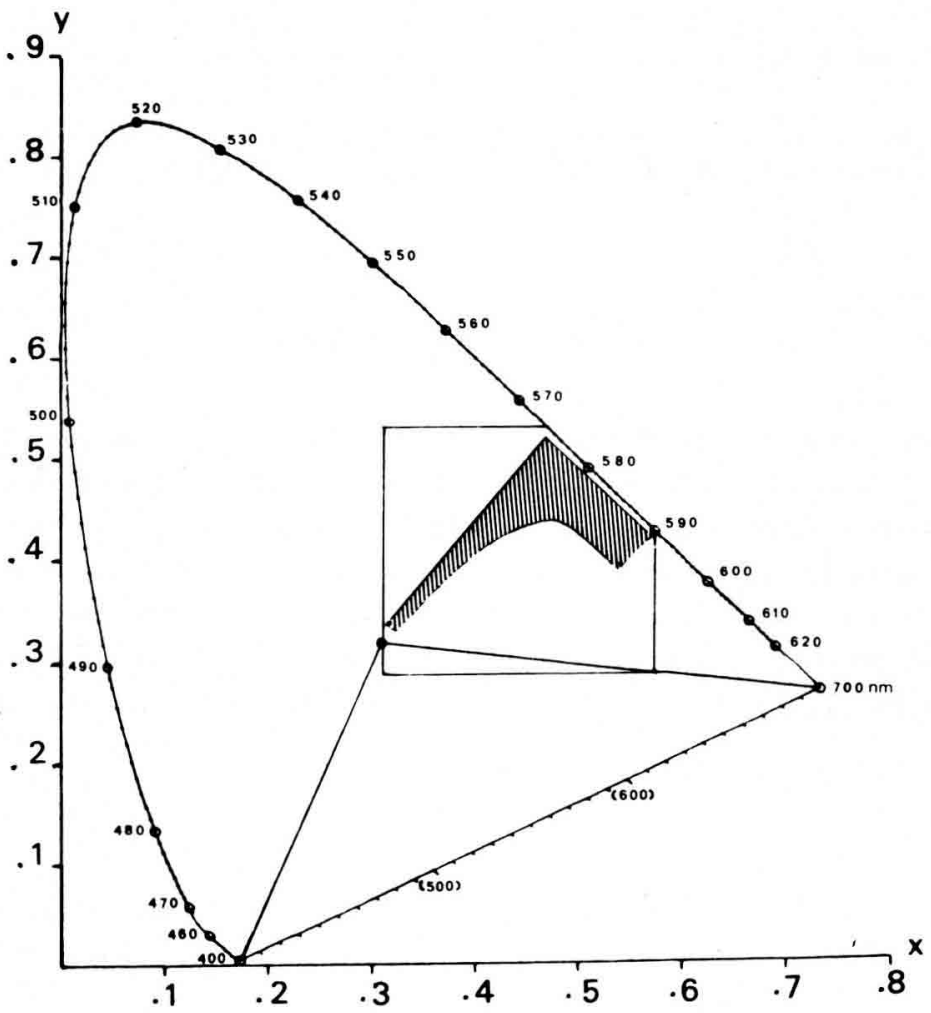

FIG 2. - Diagramme de HARDY obtenu sur table traçante.

Représentation en hachuré de la zone de couleur (mesurée en transmission) correspondant au miel en phase liquide.

FIG. 2. - HARDY diagram obtained on a plotter. Hachured area = area of color (measured in transmission) corresponding to the honey in the liquid phase.
1. Acacia
2. Romarin
3. Luzerne
4. Oranger
5. Trèfle
6. Colza
7. Tilleul
8. Lavandin
9. Tournesol
10. Pissenlit
11. Lavande
12. Thym
13. Rhododrendron
14. Bourdaine
15. Eucalyptus
16. Chataignier
17. Sapin (Jura)
18. Erica
19. Sarrasin
20. Sapin (Vosges)

La confrontation de ces indices visuels avec les mesures optiques et photo-électriques sera faite (en respectant le même ordre de rangement subjectif dans les tableaux et graphiques) pour mettre en évidence les différences avec les mesures objectives.

Les miels sont rendus parfaitement liquides et mis en cuves de verre rectangulaire. La couleur est mesurée sur un parcours optique de $10 \mathrm{~mm}$. L'appareil utilisé est un spectrophotomètre Beckmann de type D.K. 2 A à double faisceaux et enregistreur automatique en transmission (\%) ou en densite optique (Absorbance).

Le tracé des courbes s'effectue entre 350 et $700 \mathrm{~nm}$.

Le calcul conventionnel, à partir des tracés spectrographiques des valeurs chromatiques $x$ et $y$, ainsi que la projection sur des diagrammes de chromaticités, sont fait d'après les tables de HARDY (1936). On détermine ensuite la longueur d'onde dominante de la couleur d'un miel (tonalité) et le facteur de pureté 
ou saturation dans cette teinte. Le facteur luminance ou brillance du produit correspond au stimulus $Y$. Il exprime la luminosité de la coloration, d'autant plus grande que la pigmentation est faible et le produit limpide.

Sur la même série de miel, les mesures visuelles sont faites à l'aide du comparateur de LoviBond (décrit plus haut); les résultats correspondant aux indices de Pfund.

\section{IV. - RÉSULTATS OBTENUS}

La figure 3 rapporte les courbes de transmission de la lumière dans le domaine du visible et pour des miels liquéfiés. L'acacia et le lavandin sont deux produits réputés " clairs", mais on les différencie nettement par une mesure spectrophotométrique. De même des miels classés subjectivement dans la gamme des produits foncés (miels de sapin récoltés, l'un dans le Jura et l'autre dans les Vosges) se distinguent aisément par un tracé précis. Le miel de tournesol se singularise par une courbe au profil caractéristique tout à fait différente de celle de miels plus traditionnels.

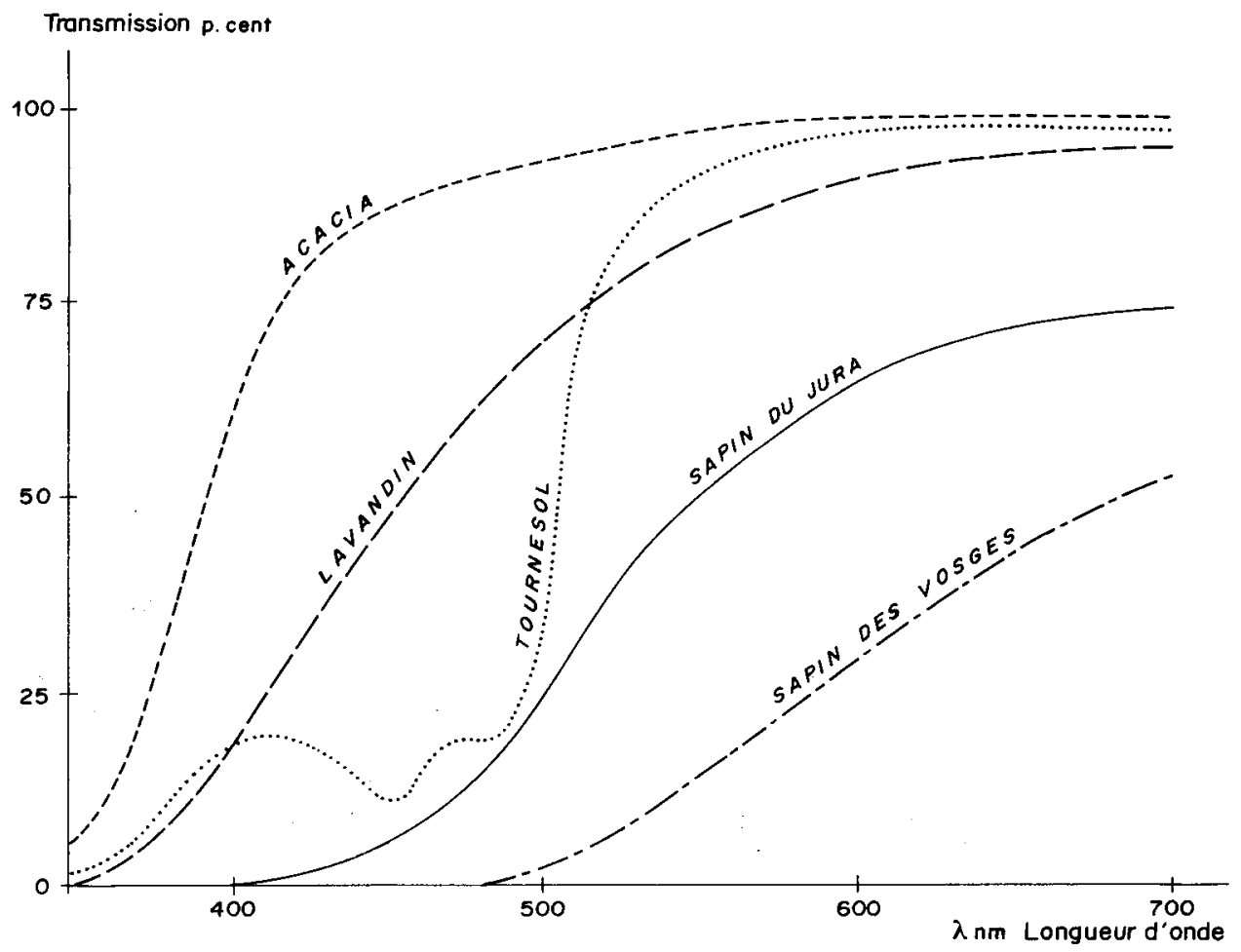

FIG. 3. - Courbes de transmission optique sur les miels liquides.

FIG. 3. - Curves of optical transmission of liquid honeys. 


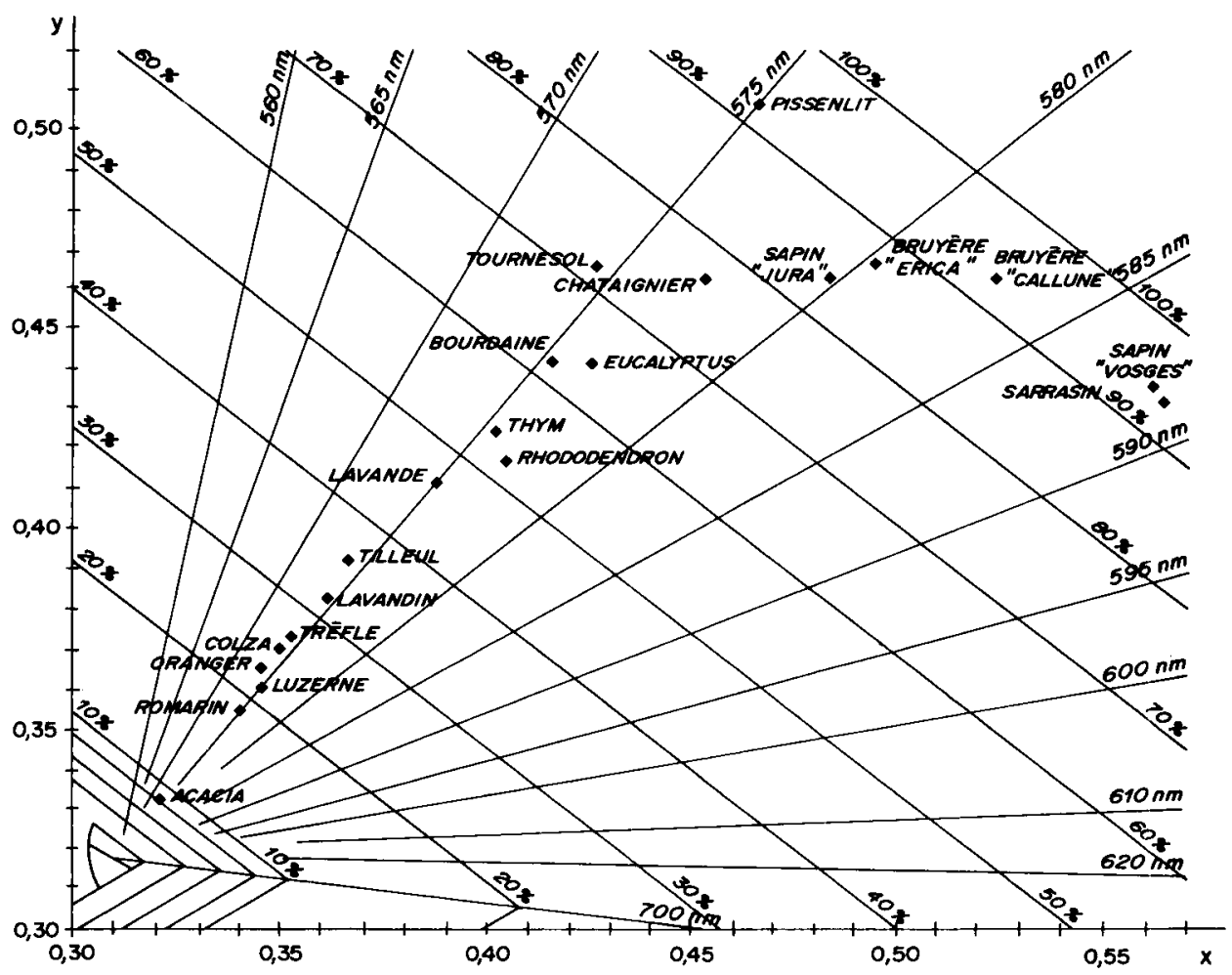

FIG. 4. - Diagramme de chromaticité des miels (système HARDY) : zone de localisation de la couleur des miels liquides.

FIG. 4. - Chromaticity diagram of honeys (HARDY system) : localization area of the color of liquid honeys.

La représentation tristimulaire des miels est limitée au plan du diagramme de chromaticité illustré à la figure 4. La plupart des échantillons se situent dans le faisceau autour d'une longueur d'onde dominante de $575 \mathrm{~nm}$. dans les chromophores ou pigments jaunes et brun clair. Les miels les plus foncés dérivent cependant vers la gamme des produits à teinte rougeâtre. La figure 5 complète cette expression qualitative de la coloration en introduisant le facteur " luminance " qui donne la notion de quantité de luminosité. Subjectivement, on a caractérisé le produit par des qualificatifs visuels très imprécis : foncé, clair, sombre et lumineux, qui sont rapportés sur le digramme. 


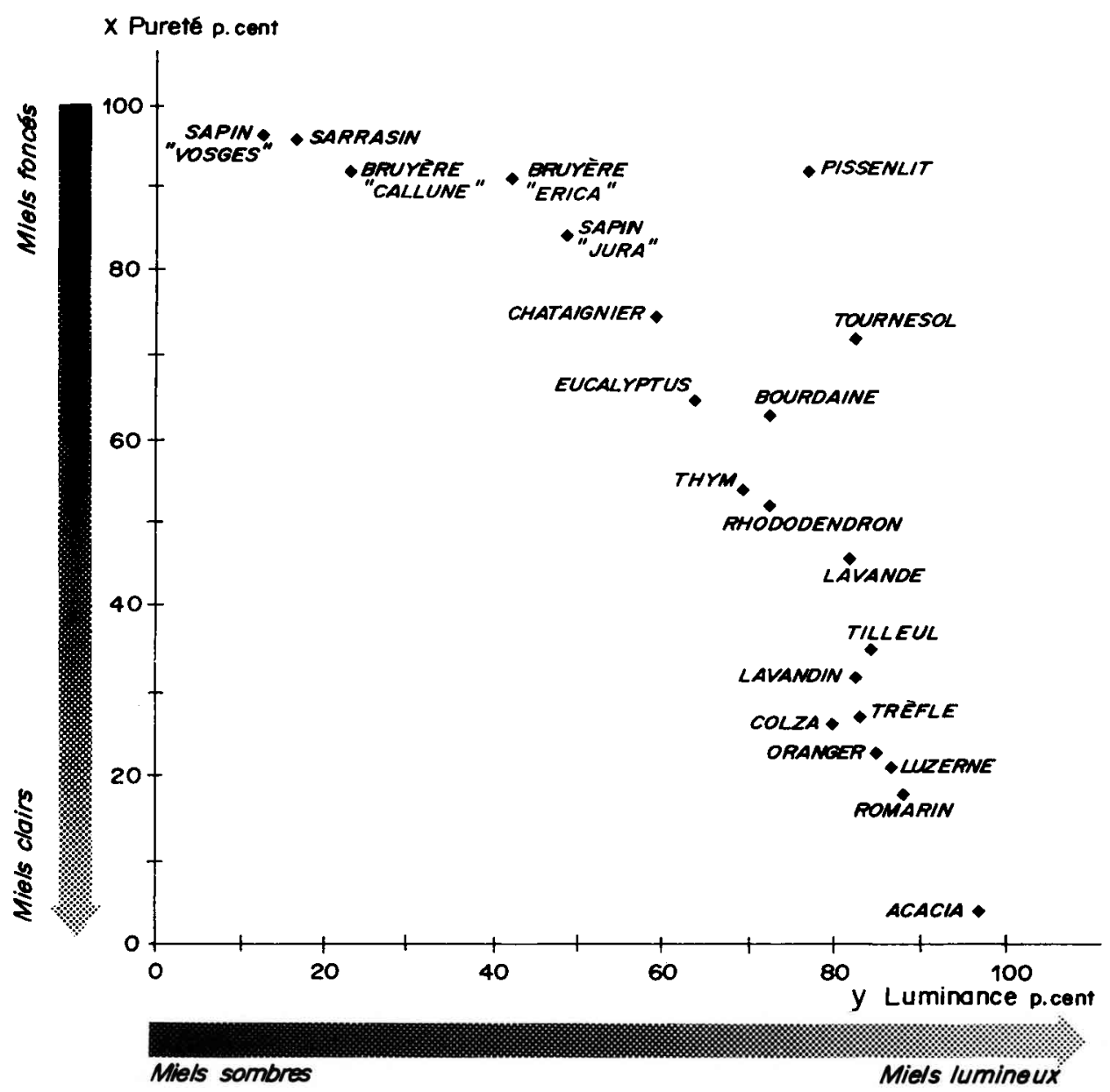

Fig. 5. - Dispersion de la couleur des miels en fonction de coodonnées chromatiques : pureté ou saturation (dans une teinte donnée) et luminance ou clarté des miels en phase liquide (dans une cuve d'épaisseur $10 \mathrm{~mm}$ ).

FIG. 5. - Color dispersion of honeys in terms of chromatic coordinates: purity or saturation (of a given shade) an brightness or clearness of honeys in liquid phase (in a $10 \mathrm{~mm}$ cell depth).

Le classement visuel, du plus clair au plus " foncé ", s'avère très insuffisant par rapport à celui, moins arbitraire, obtenu avec les indices de Pfund et surtout celui correspondant aux données réduites de la méthode spectrophotométrique (tableau 1). Ce mode d'expression, à partir des données chromatiques de la méthode tristimulaire, propose un rapport conventionnel (pureté/luminance) en corrélation évidente avec les classements subjectifs et usuels. Cette simplification discutable est néanmoins nettement plus discriminante. Elle a l'avantage de ramener l'expression 


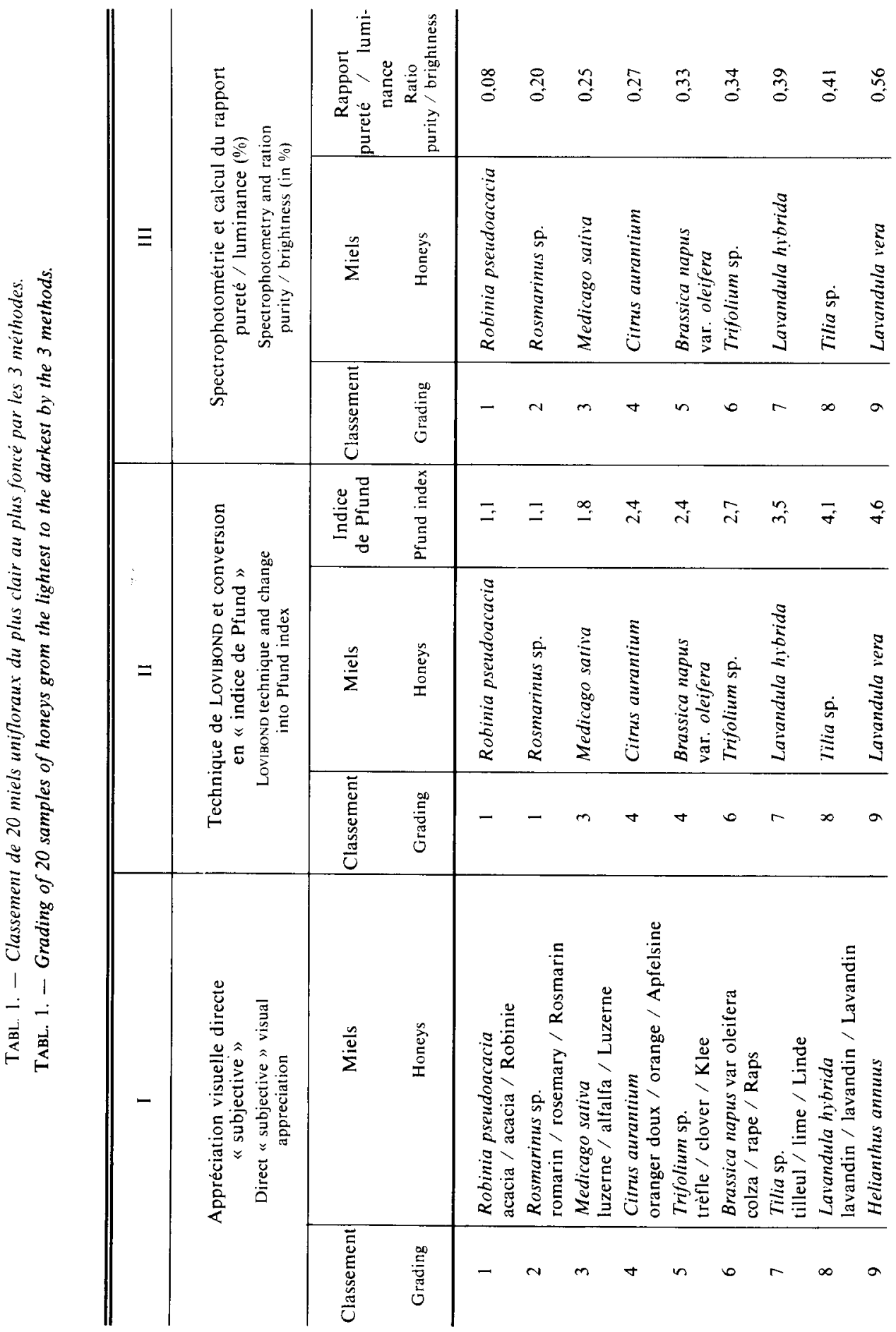




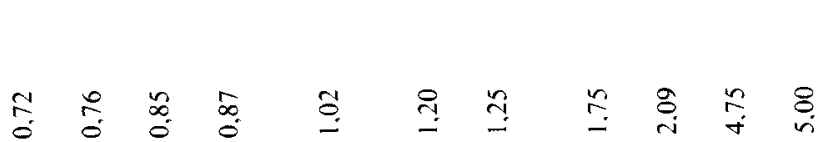

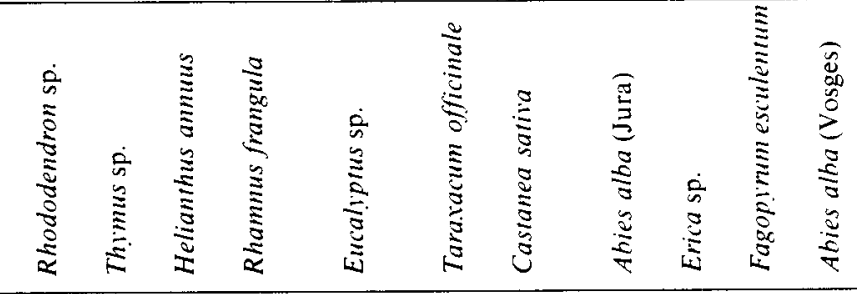

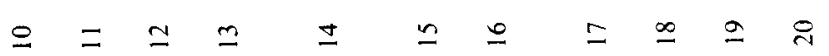

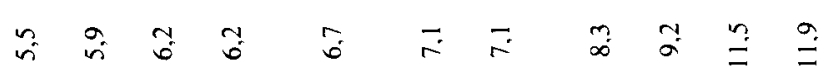

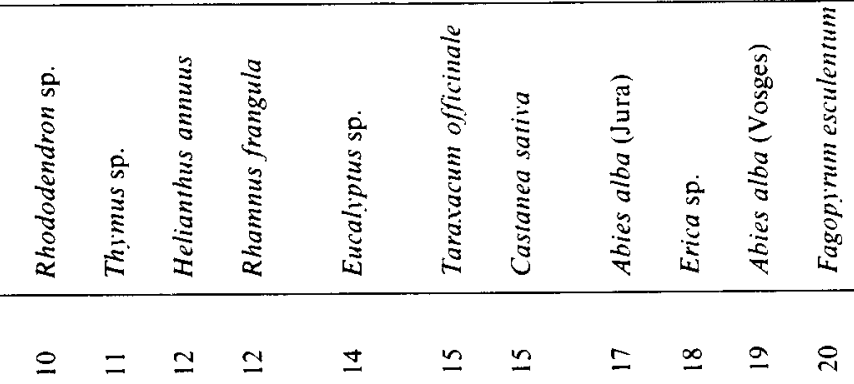

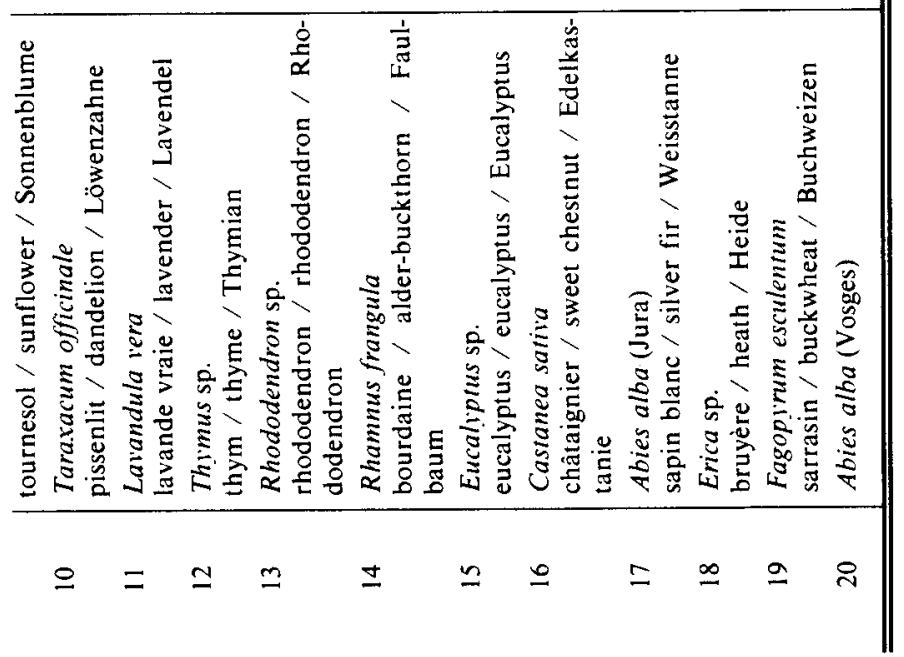


de la couleur à une seule donnée permettant un classement objectif extrêmement simple à utiliser pour les praticiens. On voit, par exemple, que les miels brillants comme le pissenlit et le tournesol apparaissent relativement clairs à l'observateur; (position 10 dans la gamme colorée pour le pissenlit) alors que leur indice de couleur les apparente plutôt à des miels foncés (position 15 pour le même miel au Pfund ainsi qu'en spectrophotométrie). Notons enfin que la détermination de l'indice du Pfund est une estimation très correcte de la couleur des miels; le classement obtenu avec cet indice est un peu moins précis mais très proche de celui découlant de l'analyse spectrophotométrique (colonnes II et III du tableau 1).

\section{V. - INTERPRÉTATION ET CONCLUSION}

La couleur est une caractéristique sensorielle importante des miels, elle varie fortement en fonction de l'origine florale des produits beaucoup plus que ne l'exprime les méthodes anciennes d'évaluation de la couleur. Les courbes de coloration réalisées sur l'ensemble du spectre de transmission de la lumière révèlent cette grande diversité aussi bien qualitativement que quantitativement. Nous avons interprété ces courbes par la méthode officielle de la C.I.E., à l'aide des paramètres conventionnels : longueur d'onde dominante, pureté de couleur et luminance. Les deux premiers apparaissent plus ou moins spécifiques de la qualité de la pigmentation et le dernier introduit une notion de quantité ou d'opacité. En utilisant ces trois paramètres, on doit pouvoir préciser de manière rigoureuse des limites de variation à l'intérieur desquelles des miels sélectionnés pourront être catalogués.

La longueur d'onde dominante est généralement stable pour la plupart des miels classiques et se situe à l'entour de $575 \mathrm{~nm}$ (ceci précise une teinte de référence utilisable dans les indices de type Pfund). Mais elle tient compte d'une manière plus spécifique des caractéristiques de pigmentation originale des miels de couleur foncé, dérivant vers $590 \mathrm{~nm}$. La même dérive de la longueur d'onde dominante s'observera sans doute pour des miels chauffés, subissant un brunissement de leur coloration naturelle initiale.

Néanmoins, cette méthodologie plus rigoureuse permet de vérifier a posteriori que les techniques de Pfund donnent des résultats acceptables et reproductibles qui sont très convenables pour la plupart des miels dans la pratique courante.

Nous révélons cependant quelques insuffisances de ce système. Par exemple, dans la gamme LOVIBOND, on ne sépare pas de manière satisfaisante des miels très clairs d'origine florale différente; de même les miels très foncés, absorbant beaucoup de lumière, sont difficiles à différencier par des comparateurs visuels.

La méthode spectrophotométrique étudiée devrait servir de système étalon pour la recherche d'une classification florale plus fine des miels. Toutefois l'indice de Pfund pourrait rester, par sa simplicité de mise en œuvre, la référence normative et commerciale. 
La technique tristimulaire pourrait permettre aussi, éventuellement, d'établir de nouvelles plaquettes ou étalons colorés mieux répartis sur l'éventail de couleur des miels, et de corriger ainsi les disques chromatiques utilisés dans les comparateurs visuels.

Retenons enfin que la perception visuelle directe des stimulus colorés est une donnée toujours entachée d'erreurs subjectives et sujette à de multiples critiques sur un plan commercial et rationnel.

En conclusion, l'extrême diversité des qualificatifs donnés par les consommateurs peuvent maintenant être repérés d'une manière très objective et précise en utilisant la méthodologie tristimulaire de la C.I.E. Une publication ultérieure montrera que cette méthode rigoureuse d'expression de la couleur sera indispensable si l'on veut, par exemple, apprécier et cataloguer des miels en phase cristallisée semi-liquides et opaques; sur ces produits, les comparateurs chromatiques sont particulièrement en défaut ou de mise en œuvre beaucoup plus difficle.

Reçu pour publication en septembre 1982

Eingegangen im September 1982

\title{
SUMMARY
}

\author{
COLOR GRADING OF HONEY
}

Color is a critical factor for the consumers'choice of honey. A unifloral honey must have a well-defined color that can be recognized and standardized by a conventional system. A mixed floral honey with a designation geographic of origin should also fall within a color range narrow enough to be recognized and be esthetically appealing.

Standard methods in general are visual and rely on more or less complicated comparators such as the Pfund color grader. The LoviBond instrument is a technical improvement based on the same principle.

The C.I.E. (International Lighting Commission) tristimulative methodology allows a much more objective approach to color grading while still taking advantage of the sensitivity of the human eye. Using this method the color of a honey is projected into a chromaticity triangle, that considers shade, tint and a coordonate which introduces the concept of color intensity and quantity.

This method can be used for clear liquid (or redissolved) or slightly turbid honey. The whole spectrographic profile of transmitted light between 350 and $700 \mathrm{~nm}$ is used.

A selction of 20 typical unifloral honey samples from commercial sources was analyzed with this method. A comparison of the results with those obtained by the classical method (Lovibon-Pfund) helps in understanding and rectifying some anomalies pointed out between an arbitrary scale and the proposed direct visual measurement of honey color. The grading of the conventional Pfund index must be kept as the commercial reference for honey colors, but the tristimulative measures are more objective and precise for identifying some kinds of honey with a particularly attractive yellow color (sunflower honey). They can also be used for establishing a new color standard. Finally, the tristimulative methodology should be used to improve the standardization of colorimetric scales used in the comparators (Lovibond) and small shade-cards plates (melloscopes).

The proposed technique of grading should make possible the definition of colors in honey that is in the process of crystallization, semi-cristallized or completely crystallized. These reflectometric analyses of surface color requires the addition of special equipment (integrating sphere) to the 
spectrophotometer. There are at present no color comparators that enable reproducible measurements to be made of liquid and turbid honey. This will be the object of a later study.

\section{ZUSAMMENFASSUNG}

\section{FARBBESTIMMUNG DES HONIGS}

Die Farbe des Honigs ist ein kritischer Faktor bei der Wahl des Verbrauchers. Ein monofloraler Honig hat eine wohl definierte Farbe in einem konventionell bekannten System, welches erlaubt, den Honig anhand seiner Farbe zu bestimmen. Ein multifloraler Honig von bekannter geographischer Herkunft sollte ebenfalls einer ausreichend schmalen Farbklasse angehören, um eine visuelle Klassifizierung zu ermöglichen.

Die klassische Technik bestand in einem mehr oder minder komplexen empirischen visuellen Vergleich mit Hilfe von Apparaten. Der bekannteste unter ihnen ist der "Pfund color grader ". Der Apparat von LoviBond ist eine technische Verbesserung dieses Apparats und basiert auf demselben Prinzip.

Die tristimuläre Methode der C.I.E. (Commission Internationale de l'Éclairage) erlaubt eine viel objektivere Farbbestimmung, die der partikulären Sensibilität des standardisierten menschlichen Auges Rechnung trägt. Diese Methode erlaubt eine Projektion der Farbe des Honigs in ein Chromatizitäts-Dreieck, das AufschluB gibt über die Farbschattierung und die Luminosität (Brillianz) und damit über die Farbintensität und -quantität.

Die vorliegenden Untersuchungen befaßten sich mit flüssigen (oder verflüssigten) Honigen, klar oder nur leicht trüb. Es wurden Daten aus dem Spektralbereich 350 bis $700 \mathrm{~nm}$ ausgewertet.

Eine Auswahl von 20 kommerziellen typischen monofloralen Honigen wurde mit dieser Methode analysiert. Die Gegenüberstellung der so erhaltenen Daten mit denen der klassischen Technik (Lovibond-Pfund) ergibt eine Erklärung und Korrektur verschiedener Anomalien zwischen einer willkürlichen Einteilung nach direkter visueller Bewertung der Honigfarbe und der Klassifizierung nach dem konventionnellen System von Pfund. Dennoch bleiben die einfachen und gebräuchlichen Techniken " a posteriori " als universelles Bewertungssystem erhalten und der Index von Pfund die kommerzielle Referenz zur Bestimmung der Farbe des Honigs. Aber die tristimulären Methoden sind objektiver und präziser bei der Identifizierung von verschiedenen Honigtypen von besonders attraktiver gelber Farbe (Sonnenblumen-Honig).

Sie könnten ebenfalls dazu dienen, neue Farbnormen für Honige aufzustellen. Schließlich könnte die tristimuläre Methodologie zur besseren Eichung der colorimetrischen Skalen für die Vergleichsapparate (Typ Lovibond) einerseits und für die Plättchen, die zur Nuancierung (im Melloscope) dienen, andererseits zur Verfügung stehen.

Die vorgeschlagene Technik der Farbbestimmung müßte eine Definition der Farbschattierung direkt am kristallisierten Zustand oder dem halb flüssigen, halb kristallisierten Zustand ermöglichen. Die reflektometrischen Analysen der Farbe der Oberfläche bedürfen eines Spezialapparates (integrierende Sphäre), der an das verwendete Spektrometer adaptiert sein muß. Keiner der existierenden Vergleichsapparate erlaubt Farbbestimmungen dieses Typs an flüssigen oder trüben Honigen, die voll reproduzierbar sind. Analysen dieser Art sollen fortgeführt werden.

\section{BIBLIOGRAPHIE}

Aubert S., 1976. - Influence de la couleur des aliments et boissons sur leur acceptabilité. Cah. Nutrition et diététique, XI (1), 15-30.

Barbier E., Valin J., 1957. - Détermination de la couleur des miels. Ann. Falsif. des Fraudes, 400-411. 
BRICE B. A. et al., 1956. - Glass color standars for extracted honey. J. Assoc. offic. Agric. chem., 11, 919-937.

HARDY A. C., 1936. - Handbook of Colorimetry, 87 p. The technology Press, Massachusetts Institute of Technology. Cambridge.

SECHRIST E. L., 1925. - The color grading of honey. U.S. Dept. of Agric., Circ. 364 (10), 1-7. 